\section{Prevalência de dor nas costas e fatores associados em escolares do Ensino Fundamental do município de Teutônia, Rio Grande do Sul}

\section{Prevalence of back pain and associated factors in elementary school students in the town of Teutônia, Rio Grande do Sul}

Matias Noll 1

Cláudia Tarragô Candotti 2

Carlos Leandro Tiggemann 3

Maira Cristina Wolf Schoenell 4

Adriane Vieira 5

\begin{abstract}
Objectives: to determine the prevalence of back pain in elementary school students in the Teutonia school district and examine the association of demographic, behavioral and hereditary factors.

Methods: a cross-section of 743 students was examined in the course of research. The data was collected from a questionnaire composed of questions about back pain over the preceding 3 months as well as demographic, behavioral and hereditary questions, which were then analyzed using multivariate analysis and prevalence ratio $(P R)$ calculations.

Results: the prevalence of back pain was $54.1 \%$ $(n=402), 17.4 \%$ of which reported that the pain made it impossible to carry out common everyday activities. Female students $(P R=1.08 ; p=0.002)$, students active in physical exercise $(P R=1.11$; $p=0.006)$ and students whose parents reported back pain $(P R=1.19 ; p=0.001)$ all had a higher prevalence of back pain.

Conclusions: the findings indicate that the high prevalence of back pain in students can be linked to gender, the practice of physical exercise and heredity. This information can be used in planning posture education programs in the Teutônia public school system.

Key words Back pain, Adolescent health Epidemiology
\end{abstract}

Programa de Pós-Graduação em Ciências do Movimento Humano. Escola de Educação Física. Universidade Federal do Rio Grande do Sul. Rua Felizardo, 750. Jardim Botânico. Porto Alegre, RS, Brasil. E-mail: matiasnoll@yahoo.com.br

\section{Resumo}

Objetivos: verificar a prevalência de dor nas costas em escolares do ensino fundamental da rede municipal de ensino do municipio de Teutônia e verificar a associação com fatores demográficos, comportamentais e hereditários.

Métodos: estudo epidemiológico transversal em que participaram 743 escolares. Os dados, coletados a partir de um questionário composto de questões sobre dor nas costas nos últimos três meses, e de questões demográficas, comportamentais e hereditárias, foram analisados a partir análise multivariável e do cálculo das Razões de Prevalência (RP).

Resultados: a prevalência de dor nas costas foi de $54,1 \%(n=402)$, sendo que destes, $17,4 \%$ referiram que esta dor impossibilitou a realização de atividades do dia-a-dia. Os escolares do sexo feminino $(R P=1,08 ; p=0,002)$, os praticantes de exercício físico $(R P=1,11 ; p=0,006)$ e os escolares cujos pais referem dor nas costas $(R P=1,19$; $p=0,001)$ apresentam maior prevalência de dor nas costas.

Conclusões: os achados indicam alta prevalência de dor nas costas em escolares, associada ao sexo, à prática de exercício físico e à hereditariedade. Informações dessa natureza poderão ser utilizadas no planejamento de programas de educação postural nas escolas municipais de Teutônia.

Palavras-chave Dor nas costas, Saúde do adolescente, Epidemiologia 


\section{Introdução}

$\mathrm{Na}$ atualidade, ocorrências de dor nas costas e de alterações posturais estão entre os principais problemas enfrentados pelos países em desenvolvimento, pois contribuem consideravelmente para limitar a vida ativa dos trabalhadores, sendo considerados problemas socioeconômicos e de saúde pública. ${ }^{1,2}$ Não obstante, esses problemas têm se manifestado também na infância e na adolescência. ${ }^{3-5}$ Paananen et al. ${ }^{6}$ verificaram que a ocorrência de dor musculoesquelética em duas ou mais áreas anatômicas é muito frequente em jovens escolares. Corroboram com esses achados os resultados de Skoffer 7 que avaliou 546 jovens de 14 a 17 anos e verificou que aproximadamente $65 \%$ deles foram acometidos por um ou mais episódios de dor durante a vida escolar.

Do mesmo modo, também tem sido relatado que a prevalência de dor nas costas em jovens escolares aumenta com o avanço da idade. Por exemplo, Ayanniyi et al., 8 ao avaliar 3185 escolares da Nigéria de 10 a 19 anos, encontraram uma prevalência de dor nas costas superior a $55 \%$ para ambos os sexos e que esta prevalência aumenta com o aumento da idade. Ainda, Shehab e Jarallah, 5 em estudo transversal realizado com 400 escolares do Kuwait, ao demonstrarem um aumento na prevalência de $31 \%$, aos dez anos de idade, para $74 \%$, aos 18 anos de idade, sugerem que este aumento gradual da ocorrência de dor pode ser devido ao aumento e ao acúmulo de sobrecarga sobre a coluna vertebral. De fato, a literatura tem evidenciado, através dos anos, e mais intensamente na última década, que a dor nas costas, independente da maneira como é reportada pelos jovens, corresponde a um problema real que acomete jovens em idade escolar.9-11

Com base nesses achados, tem sido especulado na literatura que a lombalgia na infância é preditora de lombalgia na vida adulta.7,8-11 Partindo desta premissa, três considerações podem ser realizadas: primeira, levando-se em conta que as informações sobre a prevalência de dor nas costas disponíveis na literatura não podem ser extrapoladas para diferentes contextos, devido aos aspectos sócio-culturais, ambientais e genéticos envolvidos, 3,5,6 torna-se necessário o conhecimento desta realidade e sua associação com variáveis demográficas, comportamentais e hereditárias, de cada região do país a partir da realização de estudos epidemiológicos $;{ }^{7}$ segunda, as informações fornecidas por estes estudos poderiam subsidiar o planejamento de políticas públicas de saúde e educação 3 e fomentar o desenvolvimento de Programas de Educação Postural (PEP)12,13 no ensino fundamental e médio, incentivando a prevenção da dor nas costas e das alterações da postura;4,5,11 e, terceira, considerando-se que o aprendizado e a vivência adquiridos na infância perpassam para a vida adulta, urge a inversão da lógica de uma infância com algias para uma infância saudável.11-14

Pensando na realidade local e em oferecer subsídios para o planejamento da educação física escolar, o objetivo deste estudo foi verificar a prevalência de dor nas costas em escolares do Ensino Fundamental de um município do sul do Brasil e verificar a associação com fatores demográficos, comportamentais e hereditários.

\section{Métodos}

Trata-se de um estudo epidemiológico transversal, realizado nos meses de agosto e setembro de 2011. Este estudo refere-se à primeira avaliação (linha de base) de um estudo maior, pesquisa longitudinal, em que os escolares serão acompanhados durante sete anos consecutivos do ensino fundamental ao ensino médio. Foram convidados a participar do estudo todos os 859 escolares do ensino fundamental, de $5^{\text {a }}$ a $8^{a}$ séries, de todas as escolas $(n=6)$ pertencentes à rede municipal de ensino do município de Teutônia, Estado do Rio Grande do Sul, Brasil.

Foi desenvolvido um questionário auto-aplicável com base nos questionários utilizados nos estudos de Detsch et al. 4 e Robbins et al.,15 com dez questões fechadas sobre ocorrência, frequência e intensidade da dor nas costas nos últimos três meses; de questões demográficas (idade, sexo); comportamentais (prática de exercício físico, prática competitiva de exercício físico, número de horas diárias assistindo televisão e utilizando computador, e número de horas diárias de sono) e hereditárias (ocorrência de dor nas costas nos pais).

A primeira versão do questionário foi submetida à validação de conteúdo por oito especialistas, com expertise notório conhecimento prático e de pesquisa nas áreas de postura corporal, dor nas costas e biomecânica do movimento humano que responderam a duas questões referentes à clareza, facilidade de entendimento e aplicabilidade deste instrumento. Os especialistas preencheram também uma ficha para avaliar cada questão, individualmente, nos mesmos critérios. Com base nas oito avaliações, o questionário foi modificado para sua versão final que foi novamente submetida à avaliação dos mesmos especialistas, com uma nova ficha de avaliação considerando se o questionário atendia ao objetivo da avaliação de dor nas costas e fatores associados (Tabela 1). 
Avaliação dos oito especialistas referentes à versão final do questionário.

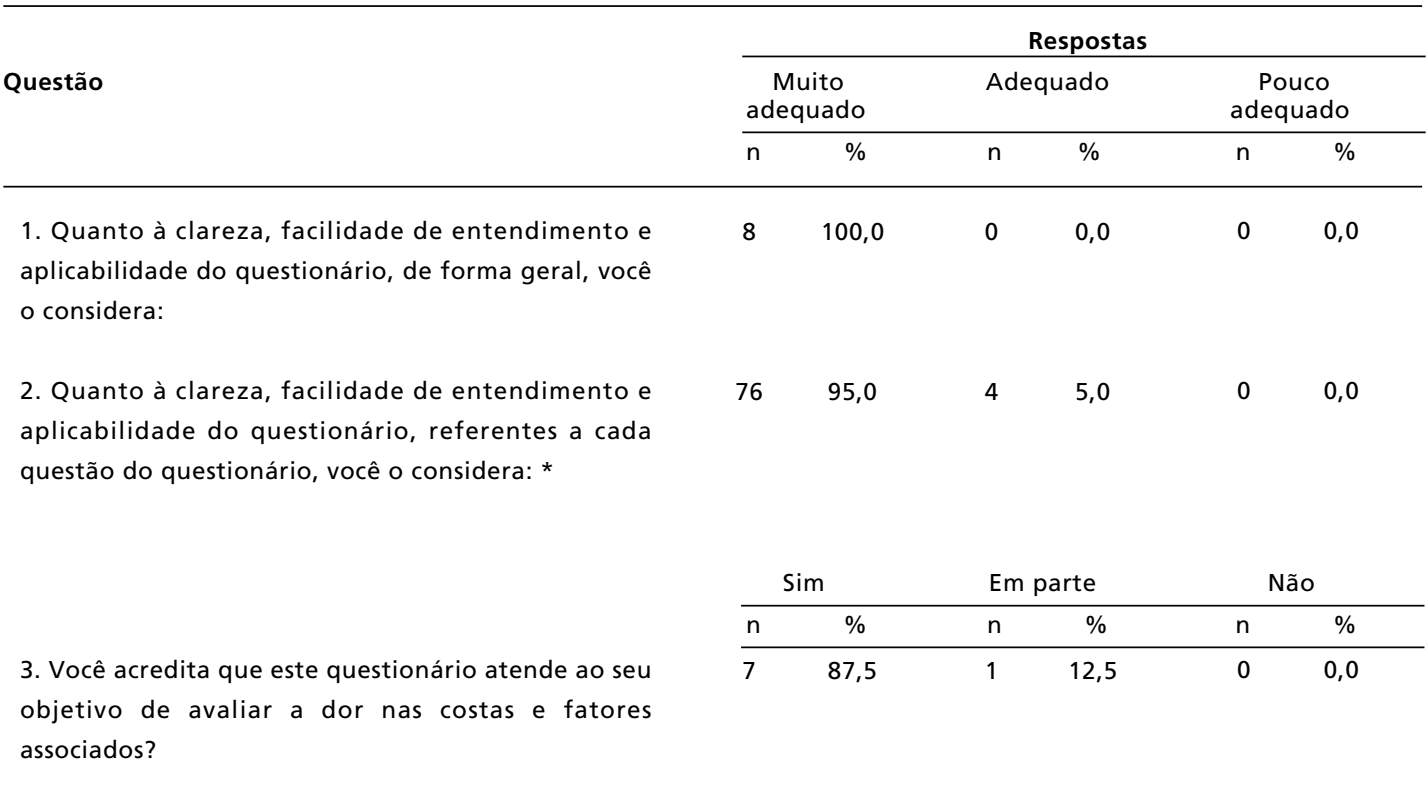

* O total de pontos desta pergunta por cada especialista é 10, sendo o máximo 80 para os 8 especialistas.

A etapa final do desenvolvimento do questionário consistiu na verificação dos índices de reprodutibilidade, ou seja, a consistência de duas avaliações (teste-reteste) dos mesmos escolares, com um intervalo de sete dias. 16 Os questionários foram aplicados em 80 escolares, de 10 a 16 anos de idade, não participantes do presente estudo. Verificou-se, por meio do coeficiente Kappa (k), que o questionário apresenta bons ( 5 questões) e excelentes (4 questões) índices de reprodutibilidade. 16

A análise dos índices de reprodutibilidade da questão 10 , referente à intensidade da dor, foi verificada por meio da Escala Visual Analógica (EVA), 15 por ser uma questão intervalar, descrita em centímetros, foi realizada a partir da Correlação de Spearmann (rs) e do teste de Wilcoxon. Os resultados referentes a este procedimento demonstraram que não houve diferença entre as respostas das duas avaliações (teste-reteste) $(p=0,349)$, e que houve alta correlação entre as avaliações ( $\mathrm{rs}=0,868, p<0,001) .16$

Os dados foram analisados utilizando o Statistical Package for the Social Sciences (SPSS) versão 18.0, a partir de estatística descritiva e do cálculo das Razões de Prevalência (RP) e seus respectivos intervalos de confiança de 95\% (IC95\%). A variável dependente foi a dor nas costas e as variáveis independentes foram: questões demográficas (idade e sexo); comportamentais (prática de exercício físico, prática competitiva ou não de exercício físico, número de horas diárias assistindo à televisão e utilizando o computador, e número de horas diárias de sono) e hereditárias (ocorrência de dor nas costas nos pais). As RP foram calculadas através de uma análise multivariada realizada a partir do modelo de Regressão de Poisson, com variância robusta. 16 Foram utilizados os testes U de Mann Whitney e Kruskal-Wallis para verificar diferenças entre os sexos e entre as idades, respectivamente, para a variável intensidade da dor $(\alpha=0,05)$.

Este estudo foi aprovado no Comitê de Ética e Pesquisa da Universidade Federal do Rio Grande do Sul, sob número 19832, e respeitou a Resolução 196/96 do Conselho Nacional de Saúde.

\section{Resultados}

Dos 859 escolares convidados a participar do presente estudo, 833 (97\%) responderam o questionário, e destes, 90 não souberam referir com clareza quanto à ocorrência ou não de dor nas costas. Desse modo, 743 (86,5\%) escolares foram incluídos na análise de dados.

A prevalência de dor nas costas nos últimos três meses referida pelos escolares foi de $54,1 \%(n=402)$. Os resultados demonstraram também que a prevalência é distinta entre escolares do sexo masculino e feminino, sendo que os percentuais de dor foram de $48,7 \%$ e de $60,1 \%$, respectivamente (Tabela 2). 
Tabela 2

Resultados da prevalência de dor nas costas nos últimos três meses e distribuição dos escolares por sexo e idade.

\begin{tabular}{|c|c|c|c|c|c|c|c|c|c|c|}
\hline \multirow{3}{*}{ Idade (anos) } & \multicolumn{5}{|c|}{ Masculino } & \multicolumn{5}{|c|}{ Feminino } \\
\hline & \multirow[t]{2}{*}{ Total } & \multicolumn{2}{|c|}{ Dor } & \multicolumn{2}{|c|}{ Sem dor } & \multirow[t]{2}{*}{ Total } & \multicolumn{2}{|c|}{ Dor } & \multicolumn{2}{|c|}{ Sem dor } \\
\hline & & $\mathrm{n}$ & $\%$ & $\mathrm{n}$ & $\%$ & & $\mathrm{n}$ & $\%$ & $\mathrm{n}$ & $\%$ \\
\hline 11 & 52 & 24 & 46,2 & 28 & 53,8 & 55 & 29 & 52,7 & 26 & 47,3 \\
\hline 12 & 94 & 41 & 43,6 & 53 & 56,4 & 89 & 57 & 64,0 & 32 & 36,0 \\
\hline 13 & 94 & 48 & 51,1 & 46 & 48,9 & 77 & 46 & 59,7 & 31 & 40,3 \\
\hline 14 & 82 & 34 & 41,5 & 48 & 58,5 & 81 & 47 & 58,0 & 34 & 42,0 \\
\hline 15 & 58 & 36 & 62,1 & 22 & 37,9 & 40 & 25 & 62,5 & 15 & 37,5 \\
\hline 16 & 12 & 8 & 66,7 & 4 & 33,3 & 9 & 7 & 77,8 & 2 & 22,2 \\
\hline Total & 392 & 191 & 48,7 & 201 & 51,3 & 351 & 211 & 60,1 & 140 & 39,9 \\
\hline
\end{tabular}

Entre os escolares que referiram sentir dor $(\mathrm{n}=402), 32,4 \%$ reportaram frequência de um episódio de dor nos últimos três meses e $39,4 \%$ um episódio mensal. Os resultados demonstraram também que $17,4 \%$ referiram que esta dor nas costas impossibilitou-os de realizar atividades do dia a dia, como brincar, estudar e praticar esportes (Tabela 3).

Tabela 3

Resultados da frequência dor nas costas nos últimos três meses e da impossibilidade de realização das atividades do dia a dia, para ambos os sexos.

\begin{tabular}{|c|c|c|c|c|c|c|}
\hline \multirow{2}{*}{ Variável } & \multicolumn{2}{|c|}{ Masculino } & \multicolumn{2}{|c|}{ Feminino } & \multicolumn{2}{|c|}{ Total } \\
\hline & $\mathrm{n}$ & $\%$ & $\mathrm{n}$ & $\%$ & $\mathrm{n}$ & $\%$ \\
\hline \multicolumn{7}{|l|}{ Frequência } \\
\hline Apenas 1 vez & 68 & 35,8 & 62 & 29,4 & 130 & 32,4 \\
\hline 1 vez por mês & 67 & 35,3 & 91 & 43,1 & 158 & 39,4 \\
\hline $1 \mathrm{vez}$ por semana & 17 & 8,9 & 21 & 10,0 & 38 & 9,5 \\
\hline 2 a 3 vezes por semana & 14 & 7,4 & 18 & 8,5 & 32 & 8,0 \\
\hline 4 vezes ou mais por semana & 12 & 6,3 & 8 & 3,8 & 20 & 5,0 \\
\hline Não souberam responder & 12 & 6,3 & 11 & 5,2 & 23 & 5,7 \\
\hline \multicolumn{7}{|l|}{$\begin{array}{l}\text { Impossibilidade de realizar } \\
\text { atividades do dia a dia }\end{array}$} \\
\hline Sim & 20 & 10,5 & 50 & 23,7 & 70 & 17,4 \\
\hline Não & 165 & 86,5 & 155 & 73,5 & 320 & 79,6 \\
\hline Não souberam responder & 6 & 3,1 & 6 & 2,8 & 12 & 3,0 \\
\hline
\end{tabular}


Os resultados referentes à intensidade da dor, descrita em centímetros, avaliada apenas nos escolares que referiram ter sentido dor nos últimos três meses, demonstraram que a mediana da intensidade da dor foi de 2,6 cm não havendo diferença entre os sexos $(p=0,381)$ e entre as idades tanto para o sexo feminino $(p=0,611)$ quanto para o masculino $(p=0,186)$ (Tabela 4).

Os resultados referentes aos fatores demográficos, comportamentais e hereditários estão apresentados na Tabela 5. A análise multivariada demonstrou que a dor nas costas está associada com as seguintes variáveis: sexo, prática de exercício físico e presença de dor nas costas nos pais.

Tabela 4

Intensidade da dor nas costas especificada por idade e sexo, conforme a escala visual analógica (EVA).

\begin{tabular}{lcccc}
\hline \multirow{2}{*}{$\begin{array}{l}\text { Idade } \\
\text { anos }\end{array}$} & \multicolumn{2}{c}{ Masculino } & Feminino \\
\cline { 2 - 5 } & $\begin{array}{c}\text { Mediana a } \\
(\mathrm{cm})\end{array}$ & $\begin{array}{c}\text { Intervalo interquartílico } \\
(25-75)\end{array}$ & $\begin{array}{c}\text { Mediana a } \\
(\mathrm{cm})\end{array}$ & $\begin{array}{c}\text { Intervalo interquartílico } \\
(25-75)\end{array}$ \\
\hline 11 & 1,95 & 2,63 & 3,1 & 3,8 \\
12 & 2,25 & 2,63 & 2,6 & 2,7 \\
13 & 2,55 & 2,3 & 2,3 & 2 \\
14 & 3,05 & 2,7 & 3,05 & 2,4 \\
15 & 2,65 & 1,27 & 2,8 & 2,3 \\
16 & 3,5 & 2,6 & 2,8 & 4,9 \\
Mediana global & $2,5 \mathrm{~b}$ & 2,2 & $2,8 \mathrm{~b}$ & 2,6
\end{tabular}

a Comparação de EVA entre idades: teste de Kruskall-Wallis $(p>0,05)$ b Comparação de EVA (Mediana Global) entre os sexos: teste de mann-Whitney $(p=0,38)$. 
Resultados de associação $\left(\chi^{2}\right)$ e razões de prevalência para a variável dor nas costas e para as variáveis demográficas, comportamentais e hereditárias.

\begin{tabular}{|c|c|c|c|c|c|c|}
\hline \multirow{2}{*}{ Variável } & \multirow{2}{*}{$\mathbf{N}$} & \multirow{2}{*}{$\%$} & \multicolumn{2}{|c|}{ Dor nas costas } & \multirow{2}{*}{$\chi^{2}$ a } & \multirow{2}{*}{ RP (IC95\%) } \\
\hline & & & $\mathrm{n}$ & $\%$ & & \\
\hline \multicolumn{7}{|l|}{ Demográficas } \\
\hline \multicolumn{7}{|l|}{ Sexo $(n=743)$} \\
\hline Masculino & 392 & 52,8 & 191 & 48,7 & 0,002 & 1 \\
\hline Feminino & 351 & 47,2 & 211 & 60,1 & & $1,08(1,03-1,13)$ \\
\hline \multicolumn{7}{|c|}{ Idade (anos) $(n=743)$} \\
\hline 11 & 107 & 14,4 & 53 & 49,5 & 0,133 & 1 \\
\hline 12 & 183 & 24,6 & 98 & 53,6 & & $1,03(0,95-1,11)$ \\
\hline 13 & 171 & 23,0 & 94 & 55,0 & & $1,04(0,96-1,12)$ \\
\hline 14 & 163 & 21,9 & 81 & 49,7 & & $1,01(0,92-1,09)$ \\
\hline 15 & 98 & 13,2 & 61 & 62,2 & & $1,08(0,99-1,18)$ \\
\hline 16 & 21 & 2,8 & 15 & 71,4 & & $1,15(1,01-1,30)$ \\
\hline \multicolumn{7}{|l|}{ Comportamentais } \\
\hline \multicolumn{7}{|c|}{ Prática de exercício físico e/ou esporte $(n=741)$} \\
\hline Não & 94 & 12,7 & 38 & 40,4 & 0,006 & 1 \\
\hline Sim & 647 & 87,3 & 363 & 56,1 & & $1,11(1,03-1,20)$ \\
\hline \multicolumn{7}{|c|}{ Prática competitiva de exercício físico $(n=642)$ b } \\
\hline Não & 358 & 55,8 & 210 & 58,7 & 0,117 & 1 \\
\hline Sim & 284 & 44,2 & 149 & 52,5 & & $0,96(0,91-1,01)$ \\
\hline \multicolumn{7}{|c|}{ Tempo assistindo televisão (horas por dia) $(n=741)$} \\
\hline 0 a 1 & 96 & 13,0 & 47 & 49,0 & 0,139 & 1 \\
\hline 2 a 3 & 246 & 33,2 & 123 & 50,0 & & $1,01(0,93-1,09)$ \\
\hline 4 a 5 & 149 & 20,1 & 85 & 57,0 & & $1,05(0,97-1,15)$ \\
\hline 6 a 7 & 76 & 10,3 & 47 & 61,8 & & $1,09(0,99-1,19)$ \\
\hline 8 ou mais & 62 & 8,4 & 40 & 64,5 & & $1,10(1,01-1,22)$ \\
\hline Depende do dia & 112 & 15,1 & 59 & 52,7 & & $1,02(0,93-1,22)$ \\
\hline \multicolumn{7}{|c|}{ Tempo utilizando o computador (horas por dia) $(n=734)$} \\
\hline 0 a 1 & 213 & 29,0 & 118 & 55,4 & 0,197 & 1 \\
\hline 2 a 3 & 211 & 28,7 & 124 & 58,8 & & $1,02(0,96-1,08)$ \\
\hline 4 a 5 & 95 & 12,9 & 54 & 56,8 & & $1,01(0,93-1,09)$ \\
\hline 6 ou mais & 52 & 7,1 & 25 & 48,1 & & $0,95(0,86-1,05)$ \\
\hline Depende do dia & 163 & 22,2 & 77 & 47,2 & & $0,95(0,89-1,01)$ \\
\hline \multicolumn{7}{|c|}{ Tempo de sono por noite (horas por dia) $(n=742)$} \\
\hline Depende do dia & 111 & 15,0 & 55 & 49,5 & 0,373 & 1 \\
\hline 0 a 6 & 75 & 10,1 & 46 & 61,3 & & $1,07(0,98-1,18)$ \\
\hline 7 & 129 & 17,4 & 73 & 56,6 & & $1,05(0,96-1,14)$ \\
\hline 8 a 9 & 324 & 43,7 & 178 & 54,9 & & $1,04(0,96-1,11)$ \\
\hline 10 ou mais & 103 & 13,9 & 50 & 48,5 & & $0,99(0,91-1,09)$ \\
\hline \multicolumn{7}{|l|}{ Hereditárias } \\
\hline \multicolumn{7}{|c|}{ Presença de dor nas costas nos pais $(n=611)$ c } \\
\hline Não & 220 & 36,0 & 85 & 38,6 & 0,001 & 1 \\
\hline Sim & 391 & 64,0 & 256 & 65,5 & & $1,19(1,13-1,26)$ \\
\hline
\end{tabular}

a Análise multivariada de cada variável no modelo. Teste Wald Chi-Square; b Somente para os escolares os quais a variável se aplica; c Foram excluídos os escolares que não tinham clareza quanto à presença ou não de dor nas costas nos pais. 


\section{Discussão}

Os resultados do estudo demonstraram que a prevalência de dor nas costas nos escolares de Teutônia encontra-se dentro das taxas descritas na literatura, que variam de aproximadamente $20 \%$ a $70 \% 6,7,17-20$ e que esta alta prevalência de dor tem imputado, para alguns escolares, na dificuldade da realização de atividades como praticar esportes, participar das atividades escolares, brincar com amigos e colaborar nos afazeres domésticos (Tabela $3)$.

Não obstante, consciente destas implicações, os resultados oriundos da análise multivariada demonstraram que a dor nas costas está associada com as seguintes variáveis: sexo, prática de exercício físico e presença de dor nas costas nos pais. Em relação aos fatores associados à dor nas costas, os resultados não possibilitam o estabelecimento de uma relação temporal de causa e efeito, devido ao tipo de delineamento utilizado, no qual as informações sobre as variáveis demográficas, comportamentais e hereditárias e a variável dependente (dor nas costas) foram obtidas ao mesmo tempo. 16

Achados similares, demonstrando maior prevalência de dor nas costas no sexo feminino, foram encontrados por Vitta et al.,17 Shehab e Jarallah $^{5}$ e Watson et al. ${ }^{3}$ Isso pode ser explicado pelo fato das meninas apresentarem, em geral, um limiar de dor inferior e características anátomofuncionais (menor estatura, menor percentual de massa muscular e massa óssea) distintas em relação aos meninos. 18 Além disso, tem sido referido que as mulheres possuem maior permissão social para expor seus sintomas e sentimentos, devendo-se ambos os eventos a fatores sociais e educacionais. 17

No que diz respeito à idade, embora os achados do presente estudo tenham demonstrado que os escolares de 15 e 16 anos apresentaram maior prevalência de dor nas costas que os escolares de 11 anos, estando de acordo com a literatura, ${ }^{9}$ a análise multivariada não os corroborou. Entretanto, Burton et al. 21 verificaram que a prevalência de dor aumentou de forma constante em cerca de $10 \%$ ao ano, ou seja, aumentando de $11,6 \%$ aos 11 anos, para $50,4 \%$ aos 15 anos.

Resultados desta natureza têm evidenciado que a lombalgia na infância pode ser considerada um fator de risco para a lombalgia na vida adulta,7,9-11 bem como de que o aumento gradual da prevalência de dor com a idade pode ser resultante do acúmulo de sobrecarga sobre a coluna vertebral ao longo dos anos, como por exemplo, no transporte de mochilas e outros objetos pesados e de modo assimétrico, e na permanência por longos períodos na posição sentada, entre outros.5,21

A literatura tem sugerido que a atividade esportiva, recreativa 6,17 e competitiva, ${ }^{22}$ são fatores que podem aumentar o risco de dor nas costas na infância e adolescência. Tal fato pode ser justificado por um conjunto de variáveis, tais como o tipo de esporte, o nível de exigência, a intensidade do treinamento e o grau de trauma agudo gerado pelo esporte, principalmente em crianças e adolescentes. 3,17 No entanto, os resultados deste estudo não são conclusivos, uma vez que não foram investigados a associação entre prevalência de dor nas costas e fatores como o tipo de esporte, a frequência de prática, a intensidade do treinamento, demonstrando uma fragilidade no instrumento de avaliação.

Ainda quanto aos fatores associados à dor nas costas, os resultados corroboram com Masiero et $a l ., 20$ os quais também encontraram relação entre a dor nas costas de pais e filhos. Embora a razão desta relação ainda permaneça obscura, especula-se que isto possa ocorrer por questões de hereditariedade ou por fatores comportamentais e psicológicos, ou seja, os jovens que convivem com pais que se queixam de algias na coluna vertebral podem estar inclinados a responder também que sofrem de dor nas costas. 20

Embora no presente estudo não tenha ocorrido associação entre o tempo assistindo televisão e utilizando o computador por dia, a literatura tem referido que o tempo de permanência na posição sentada é um fator de risco à prevalência de dor nas costas. Por exemplo, Vitta et al.17 relataram que os escolares que assistiam televisão acima de duas horas diariamente, apresentavam mais chances de ter dor nas costas. Considerando que, aproximadamente, $70 \%$ dos escolares de Teutônia assistem televisão por mais de duas horas diariamente e permanecem em média de quatro a cinco horas diárias na posição sentada durante os períodos regulares de ensino, 23 especula-se que mais de $60 \% 17$ deles permaneçam no mínimo oito horas diárias em uma posição sentada, que além de ser caracterizada como uma atividade sedentária, é um fator de risco para a ocorrência de dor nas costas 17 e de alterações posturais. $2,4,5$

Por fim, conclui-se que os escolares de Teutônia apresentam alta prevalência de dor nas costas, sendo maior nas meninas em comparação aos meninos, nos praticantes de exercício físico, e naqueles em que os pais apresentam dor nas costas. Os resultados encontrados e aqueles descritos na literatura sugerem que, independentemente do país, crianças e adolescentes inseridos no contexto escolar estão sujeitos ao desenvolvimento de dor nas costas, fato esse 
refletido na alta prevalência de dor nas costas desta população. Algumas das diferenças entre os estudos quanto à prevalência de dor nas costas e aos fatores de risco associados devem-se, possivelmente, aos diferentes desenhos dos estudos (transversal ou longitudinal), ao método de coleta de dados, à qualidade dos instrumentos de avaliação e ao conceito de dor nas costas. 17,20 Não obstante, independente-

\section{Referências}

1. Karahan A, Bayraktar N. Determination of the usage of body mechanics in clinical settings and the occurrence of low back pain in nurses. Int J Nurs Stud. 2004; 41: 67-75.

2. Candotti CT, Noll M, Cruz M. Prevalência de dor lombar e os desequilíbrios musculares em manicures. Rev Arq Movimento. 2010; 6: 125-40.

3. Watson KD, Papageorgiou AC, Jones GT, Taylor S, Symmons DPM, Silman AJ, Macfarkane GJ. Low back pain in schoolchildren: occurrence and characteristics. Pain. 2002; 97: 87-92.

4. Detsch C, Luz AMH, Candotti CT, Scotto de Oliveira D, Lazaron F, Guimarães LK, Schimanoski P. Prevalência de alterações posturais em escolares do ensino médio em uma cidade no Sul do Brasil. Rev Panam Salud Publica. 2007; 21: $231-8$.

5. Shehab DK, Jarallah KF. Nonspecific low-back pain in Kuwaiti children and adolescents: associated factors. J Adoles Health. 2005; 36: 32-5.

6. Paananen MV, Taimela SP, Auvinen JP, Tammelin TH, Kantomaa MT, Ebeling HE, Taanila AM, Zitting PJ, KArppinen JI. Risk factors for persistence of multiple musculoskeletal pains in adolescence: A 2-year follow-up study. Eur J Pain. 2010; 14: 1026-32.

7. Skoffer B. Low Back Pain in 15 to 16 year old children in relation to school furniture and carrying of the school bag. Spine. 2007; 32: 713-7

8. Ayanniyi O, Mbada CE, Muolokwu CA. Prevalence and Profile of Back Pain Nigerian Adolescents. Med Princ Pract. 2011; 20: 368-73.

9. Jeffries LJ, Milanese SF, Grimmer-Somers KA. Epidemiology of Adolescent Spinal Pain: A Systematic Overview of the Research Literature. Spine. 2007; 32: 2630-7.

10. Trevelyan FC, Legg SJ. Back pain in school children: where to from here? Appl Ergon. 2006; 37: 45-54.

11. Steele EJ, Dawson AP, Hiller JE. School-based interventions for spinal pain. Spine. 2006; 31: 226-33.

12. Candotti C, Rohr J, Noll M. A educação postural como conteúdo curricular da educação física no ensino fundamental II nas escolas da cidade de Montenegro/RS. Movimento. 2011; 17: 57-77

Recebido em 13 de dezembro de 2011

Versão final apresentada em 22 de agosto de 2012

Aprovado em 26 de setembro de 2012 mente da natureza dos estudos, todos refletem a realidade dos escolares, a qual deveria ser revisitada por uma articulação entre as políticas públicas de saúde e educação. O desenvolvimento de ações conjuntas destinadas a promoção e prevenção da dor nas costas da população de escolares, como por exemplo, com a realização de PEP, possibilitaria educá-los já nos primeiros anos do ensino fundamental.12,14,24
13. Candotti CT, Roth E, Noll M. Evaluation of weight and mode of transport of student in school of education. Rev Paul Pediatr. 2012; 30: 100-6.

14. Candotti CT, Nunes SE, Noll M, Freitas K, Macedo CH. Efeitos de um programa de educação postural para crianças e adolescentes após oito meses do seu término. Rev Paul Pediatr. 2011; 29: 577-83.

15. Robbins M, Johnson IP, Cunliffe C. Encouraging good posture in school children using computers. Clinical Chiropractic. 2009; 12: 35-44.

16. Pestana MH, Gageiro JN. Análise de dados para Ciências Sociais. Lisboa: Sílabo; 2003.

17. Vitta A, Martinez MG, Piza NT, Simeão SFA, Ferreira NP. Prevalence of lower back pain and associated factors in students. Cad Saúde Pública. 2011; 27: 1520-8.

18. Ferreira GD, Silva MC, Rombaldi AJ, Wrege ED, Siqueira FV, Hallal PC. Prevalence and associated factors of back pain in adults from Southern Brazil: a population-based study. Rev Bras Fisio. 2011; 15: 31-6.

19. Zapata AL, Moraes AJP, Leone C, Doria-Filho U, Silva CAA. Pain and musculoskeletal pain syndromes related to computer and video game use in adolescents. Eur J Pediatr. 2006; 165: 408-14.

20. Masiero S, Carraro E, Celia A, Sarto D, Ermani M. Prevalence of nonspecific low back pain in schoolchildren aged between 13 and 15 years. Acta Paediatr. 2008; 97: 212-6.

21. Burton AK, Clarke R, McClune T, Tillotson K. The natural history of low back pain in adolescents. Spine. 1996; 21 : 2323-8.

22. Hangai M, Kaneoka K, Okubo Y, Miyakawa S, Hinotsu S, Mukai N, Sakane M, Ochiai N. Relationship between low back pain and competitive sports activities during youth. Am J Sports Med. 2010; 38: 791-6.

23. Freire IA, Teixeira TG, Sales CR. Hábitos posturais: diagnóstico a partir de fotografias. Conexões. 2008; 6: 28-41.

24. Candotti CT, Lemos AP, Noll M. Escola postural para crianças de 10 a 14 anos inserida no contexto do ensino fundamental. Rev Bras Ciên Mov. 2011; 19: 33-44. 